\title{
Educación Como Proceso Dialógico Donde El Sujeto Es EI Principal Agente De Cambio
}

\section{Education As A Dialogic Process Where The Subject Is The Main Agent Of Change}

\section{(1) Hermencia Rocio Pico \\ (D) Dora Inés Arroyave Giraldo 2}

Recepción: Abril 14 de 2021

Aprobación: Mayo 15 de 2021

Publicación: Junio 30 de 2021

Cómo citar este artículo: Pico, Hermencia. Arroyave G, Dora (2021). "Educación como proceso dialógico donde el sujeto es el principal agente de cambio".

Miradas, Vol. 16, $\mathrm{N}^{\mathrm{o}}$ 1.pp. 97 - 118 https://doi.org/10.22517/25393812.24863

\section{Resumen}

El presente documento tiene como objeto ofrecer una mirada frente a la educación como proceso dialógico que concibe al sujeto como agente de cambio y transformación de la realidad social, política y cultural; en consecuencia, se llevó a cabo una revisión crítica de literatura sobre currículo, educación popular y complejidad, pensados como partes de un todo llamado educación. Así, se aborda el currículo pensado como eje organizacional de la educación que procura generar propuestas contextualizadas, provoca el repensar la escuela en la riqueza de las experiencias que otorgan las vivencias de los alumnos y

1 Magister en Educación. Docente de Educación Básica Primaria. Colegio Integrado Pedro Santos -Pinchote, Santander. Candidata a doctora en Ciencias de la Educación de la Universidad de San Buenaventura-Medellín. ORCID: 0000-00017063-1404 hermencia14@gmail.com

2 Doctora en Educación. Profesora investigadora de la facultad de educación de la Universidad de San BuenaventuraMedellín. Colombia. Líder del grupo de investigación ESINED. Estudios Interdisciplinarios Sobre Educación. Líder de la línea: Estudios Críticos Sobre Educación y Currículo. ORCID: 0000-0003-0913-4841 dora.arroyave@usbmed.edu.co; diarroyave@hotmail.com 
sus contextos, y se fortalece en la visión del docente cosmopolita que está en constante reflexión de su práctica.

Además, en la educación popular se halla una iniciativa pensada para liberar al ser humano de los problemas y fenómenos creados por los procesos de desarrollo que colonizan, alienan y persuaden en favor de la globalización. Así mismo, se señala el alcance de la complejidad en la educación desde la comprensión del conocimiento dinámico, acompañado de las paradojas como un elemento para fortalecer la educación en la visión de cambio, el fomento de la crítica en los docentes y estudiantes y una forma de robustecer la dialógica en las aulas. Se concluye que la educación es un proceso dinámico, cambiante y emancipador, sobre el cual se reflexiona a partir de diferentes posturas, nociones, modelos o elementos que lo enriquecen y permiten repensarlo desde la capacidad crítica de los sujetos.

Palabras clave: Complejidad, Currículo, Educación, Educación Popular, Proceso de enseñanza-aprendizaje.

\section{Abstract}

The purpose of this document is to offer an overview concerning education as a dialogic process that conceives the subject as a change agent and transformation of social, political, and cultural reality. Hence, a critical revision of literature about curriculum, popular education, and complexity was carried out, always thinking of them as a whole called education. Thus, the curriculum is approached as an organizational axis of education that seeks to generate contextualized proposals, causing rethink the school in the experiences' wealth that the students' experiences grant and their context, strengthening the cosmopolitan vision of the professor, who is in constant reflection about his/her practice.
On the other hand, in popular education, there is an initiative conceived to release the human being of the problems and phenomena created by the development processes that colonize, alienate, and persuade in support of globalization. Moreover, the scope of the complexity in education is pointed out from the understanding of the dynamic knowledge, matched of the paradoxes as an element to strength education in the vision of change, the promotion of the critical in the teachers and students, and a way to reinforce the dialogic in the classrooms. It concludes that education is a dynamic process, changing, and emancipatory, about which is reflection as of different positions, notions, models, or elements that enrich it and allow rethink it from the critical capability of the subjects.

Keywords: Complexity, curriculum , Education, Popular education, teachinglearning process.

\section{Introducción}

Los momentos de crisis producen escenarios para nuevas tomas de decisiones y ponen en evidencia problemas que permanecían ocultos a ojos de la mayor parte de la ciudadanía (Porlan, 2020, p. 1502-1)

En diferentes escenarios de disertación, la educación es pensada como medio para generar conocimiento y desarrollar en los sujetos habilidades comunicativas, de pensamiento y capacidad crítica, logrando un estado de madurez materializado en procesos metacognitivos, que le lleven a la reflexión sobre sí mismo y a pensar en beneficio de otros y los requerimientos de su contexto; desde esta perspectiva, la educación privilegia el diálogo de saberes que transita en diferentes nociones o concepciones vinculadas 
a su sentido de proceso, de ahí la importancia de discutir respecto a la incidencia que ejercen en la educación algunos elementos como el currículo, la educación popular y la complejidad. Dichos elementos, se desarrollan a partir de tres apartados que configuran el documento.

El primer apartado "la educación con propósito humano", compara concepciones de currículo como elemento de reflexión, generador de procesos de cambio y apertura en las escuelas, como lo indican Hansen (2013) y Giraldo, et al., (2019), frente a otras realidades de este, en función de lo administrativo, constituido por acciones repetitivas con tendencia homogeneizadora del proceso de enseñanza y aprendizaje. Así, se amplía la visión de currículo como medio para enriquecer las aulas en el reconocimiento de nuevos modelos pedagógicos, en la generación de experiencias significativas surgidas del contexto y en la apertura al cambio del profesor cosmopolita (Sánchez y Gracia, 2017; Hansel, 2013, Beltrán y Cuellar, 2014).

El segundo apartado titulado "En busca de sociedades más justas: proyecto pedagógico de educación popular", se reconoce la educación popular como propuesta político-pedagógica, en la que docente $y$ estudiante construyen aprendizajes significativos a partir de sus interacciones $y$ en acciones contextualizadas pensadas para emancipar la sociedad de los problemas que le afectan en forma masiva (Mejía, 2014; Muñoz, 2013; De la Calle, 2011). Además, se entretejen apuestas de la educación popular para el cierre de brechas sociales y territoriales a partir del diálogo y confrontación de saberes, que son la principal herramienta del educador popular.

Por último, el apartado " $L a$ complejidad y sus elementos para la educación", prioriza algunos aspectos complejos como las paradojas y la creación de redes de conocimiento, que han permitido avances significativos en el proceso educativo, centrados en definición de espacios de reflexión y crítica, donde la duda, las incertidumbres y las situaciones problemas son ejes de navegación en la construcción de conocimiento dinámico y dialógico.

Finalmente, el artículo compila en las conclusiones los hallazgos del recorrido hecho sobre los textos revisados que permiten la configuración del escrito y los aportes que desde el punto de vista de las autoras sostienen como importantes para el lector, función de pensar la educación como proceso dialógico donde el sujeto es el principal agente de cambio.

\section{Educación con propósito más humano}

La humanidad transformada por fin en Humanidad, la nueva comunidad englobadora de la Tierra-patria y la metamorfosis de la humanidad son las caras de la nueva aventura humana posible. (Morin, 2020, p. 85)

\section{El currículo pensado no solo para la administración}

La idea de estructura organizacional ha permeado todos los campos del saber, y allí se han generado un sinnúmero de epistemologías, 
paradigmas y concepciones conceptuales alrededor de esta y la educación no es ajena a esto, ya que sin la organización estructural del sistema educativo y de las instituciones en sí, es posible que no se dé un proceso de enseñanza $y$ aprendizaje organizado o jerárquico como el Estado propone desde sus lineamientos y orientaciones generales; dicha posibilidad, suscitaría un caos frente a la zona de confort de quienes piensan solo en cumplir con los mínimos que orientan las políticas educativas, lo cual no es conveniente para las masas de poder.

En este orden de ideas, la educación tiene el currículo como eje organizacional, reconocido generalmente como un mecanismo estructural de conceptos, áreas, estrategias, modelos pedagógicos $\mathrm{y}$ esquemas administrativos, entre otros, que orientan una institución y en el cual se desarrollan acciones que, por ser lineales para su cumplimiento, sesgan el fundamento, interpretación y uso de otras opciones pedagógicas que enriquecerían dicho eje. Así pues, un eje problematizador en la concepción de currículo "es su dependencia organizativa, su vinculación a formas de administración de un sistema, mezclándose, o confundiéndose, o justificándose mutuamente las razones administrativas con las educativas" (Contreras 2010, p. 549).

No obstante, esta idea de currículo puede cambiar para pensarse en un medio que enriquece los procesos educativos y emancipa la educación de las políticas alienantes y globalizante, dando paso a posturas más críticas sobre la importancia de este, como se concibe a continuación:

El currículo no es un concepto abstracto que exista por fuera de la experiencia humana $o$ un documento ingenuo o neutro; es una forma de organizar un conjunto de prácticas educativas que tienen lugar en un tiempo y espacio determinados y con personas concretas (Giraldo, et al., 2019, p. 12).

Dicho de otra manera, el currículo para la educación debe cimentarse en la realidad los actores educativos, pensado como ejercicio dinámico y cambiante, que se construye y reconstruye a partir de las experiencias que brinda cada individuo, su contexto local y lo que subyace de planos globales. Por consiguiente, quienes diseñan este elemento deben estar dispuestos al cambio, abrirse a nuevas posibilidades y navegar en aguas que les permita enriquecer sus prácticas educativas, así lo afirma Hansen (2013), al concebir la idea de "currículo como una respuesta viva ante la experiencia: como una expresión de los intentos por producir sentido, por entender, por apreciar, por sentirse como en casa" (p.113).

Esta mirada posibilita inferir que detrás de cada experiencia educativa hay un proceso de reflexión sobre los individuos que son afectados por el currículo, a fin de facilitar su bienestar sin limitar su imaginación, generando en ellos una visión de educación más allá de un sistema que educa desde áreas para un rol específico; siendo 
vista, como un medio para transformar vidas, dar la oportunidad de generar inquietudes, despejar incertidumbres y edificar procesos de investigación que susciten otros modos de repensar la educación y la coexistencia para que los seres humanos sean más humanos. Para esto, necesitamos ampliar el campo visual del currículo, sería pensar en "una mayor apertura institucional, de tal manera que puedan proliferar distintas formas de concebir las propuestas de enseñanza y los encuentros educativos que puedan ocurrir en su seno" (Contreras 2010, p.565).

A la idea anterior, Giraldo, et al., (2019), añaden una concepción de currículo como "sistema abierto que permite modificaciones internas y externas y que ve el cambio como un asunto transformativo y no progresivo" (p. 13). Esto indica que, frente a las nuevas miradas educativas, el currículo no solo es un documento en ejercicio; por el contrario, puede configurarse como un todo de las partes que conforman la educación, convirtiéndose en pilar de un engranaje que se transforma a medida que lo requiere, mejorando, cambiando o reubicando piezas tras las posibilidades que ofrece el contexto de cada institución educativa, y dando paso a un currículo transformado en legado cosmopolita que, para Hansen (2013) es una orientación por derecho propio respecto a la creatividad cultural, entendida en su sentido sociolingüístico, centrado en la práctica grupal e individual, donde la escuela se vislumbra como un espacio de reflexión, de reconfiguración de culturas, cosmovisiones e ideas, que al ponerse en diálogo conlleven a mejores aprendizajes en los estudiantes y a la construcción de ciudadanos más humanos y consientes del medio en el que sobreviven.

Por lo anterior, debe existir una postura de oposición a mantener una visión única del currículo; de lo contrario se recaería en el ideal de currículo secuenciado, que en palabras de Contreras (2010), es aquel cuyos "contenidos correspondientes a cada nivel tienen que distribuirse con precisión a lo largo del curso, fijándose un mismo ritmo de aprendizaje para todos" (p. 555). Este currículo alimenta la idea de una educación homogenizada, sin tener en cuenta escenarios reales donde no todos los seres aprenden a un mismo ritmo ni de la misma forma; por lo tanto, la tarea educativa es la reflexión continua del currículo pensado como un medio transformador, en el que intervienen para su definición los diferentes actores educativos, así como el reconocimiento de la diversidad de saberes y requerimientos de los contextos permeados por el proceso educativo.

\section{Atreverse a explorar para repensar la educación}

Desde la cumbre de las ideas aristotélicas, el emerger de nuevos escenarios para la enseñanza como los ofrecidos por Pestalozzi, Dewey, Decroly y Montessori, entre otros grandes de la pedagogía, hasta el abrir campo a elementos de la complejidad, la educación ha sido repensada por el hombre en busca de fortalecer su capacidad de aprender y enseñar, de avanzar, detenerse y volver a iniciar. Sin embargo, en la actualidad se aprecia una tendencia hacia una educación más 
estática, que repite las mismas acciones y lleva un orden de enseñanza alrededor de normativas educativas en las que se proyectan aulas pensadas como "una secuencia de actividad que determina un currículum fragmentado por cursos y materias, y un horario y un calendario que establecen un momento y un tempo para el aprendizaje académico, separado a su vez de otras experiencias y saberes" (Contreras 2010, p. 551)

Es así que, la educación ha venido cruzando aguas revueltas entre nuevas concepciones de la pedagogía hasta el anclaje de modelos como el de la escuela graduada, que se ha convertido en modelo usado por gran parte de instituciones educativas, debido a los beneficios que se han visto sobre el mismo en cuanto constituyó una nueva forma de reorganizar la educación; no obstante, ha recibido fuertes críticas alrededor de convertir el currículo, la práctica y la experiencia de enseñanza aprendizaje en un espacio poco dinámico; esta idea es coherente con lo expresado por Contreras (2010),

Una escuela, por lo demás, que se ha convertido en un engranaje de un monstruo gigantesco, el "sistema educativo", lleno de burocracia, de controles, de razones administrativas más que pedagógicas para infinidad de sus prácticas, que basa el funcionamiento de su maquinaria en la lógica de la normativa (que busca el obligado cumplimiento al margen de las personas implicadas), más que en la de la relación, que es el espacio para encontrar lo adecuado. (p. 552)

Como se ha dicho, la escuela graduada es un modelo muy usado, que ha sido censurado; sin embargo, se mantiene en pie porque fluye sin presiones, sus actores se habitúan a las implicaciones de esta y porque la mayoría de acciones ya están diseñadas, conociéndose la ruta de su ejecución sin pensar en otras necesidades; en contraposición, Sánchezy García (2017) indican que "si no hay transformación no hay educación, necesitamos una educación que contribuya a cambiar el mundo, humanizándolo" (p. 3). Con esto se especifica que se requiere de una escuela pensada para los seres que forma y se forman, teniendo en cuenta sus exigencias, posibilidades y territorios en el que están inmersos, lo cual afecta sus intereses, sus ideales y por tanto, permea el proceso educativo.

De ahí que se piense en una escuela cuya "educación favorezca la aptitud natural del espíritu para plantear y resolver los problemas y correlativamente estimular el pleno empleo de la inteligencia general" (Morin, 2002, p. 24), una escuela así, consciente que los estudiantes logren poner en duda o refutar ideas expuestas, procura procesos para fortalecer la creatividad y genera espacios de argumentación y debate para los educandos dialoguen sobre lo que les es propio en sus contextos y las realidades mostradas en escenarios globales. En este sentido la escuela alcanza una organización del conocimiento desde la cual se interrelacionan los saberes, las culturas y los individuos para formar 
parte de un todo y así dejar emerger un pensamiento "ecologizante" como el propuesto por Morin (2002), que reconoce la diversidad, busca interacciones entre todo fenómeno y su contexto y permite pensar local y globalmente.

En la misma línea de ideas expuestas, Beltrán y Cuellar (2014) mencionan que "el fin inmediato de la educación es el desarrollo de la capacidad para transformar el medio, superando las miradas que concebían el ambiente como algo fijo que proporcionaría la norma de los cambios en el organismo" (p.166). En esta perspectiva, los actores de la educación están llamados a construir en la experiencia y la experimentación este proceso, como lo han hecho los pioneros de modelos como el de Montessori o el de la pedagogía activa, que rompen con paradigmas de la escuela tradicional. Estos modelos circulan alrededor de "otras escuelas", que para Contreras (2010) son "un fenómeno concreto: la existencia de escuelas que no se han configurado por los patrones que tenemos asociados a lo que es una escuela" (p. 550).

En lo mencionado, las llamadas "otras escuelas" invitan a avivar las experiencias, convirtiéndolas en procesos significativos para el aprendizaje, acompañadas de ambientes generadores de pretextos donde los estudiantes exploran, indagan y enriquecen sus saberes a partir de las interacciones con los demás. Así, estas otras escuelas cohabitan con modelos como el de "escuela graduada" y proyectan sus resultados con el propósito de invitar a las instituciones educativas a que las exploren, reconociendo en ellas su centro de aprendizaje en los niños y niñas quienes:

Pueden atender al sentido íntimo de sí, en relación a los demás, y en donde la tarea siempre en construcción (mientras se hace y se vive todo lo demás) es, en el fondo, la exploración de la libertad, entendida como la exploración y la apertura de sí a lo otro (Contreras, 2010, p. 553).

Teniendo en cuenta lo anterior, se deduce que al individuo en formación hay que enseñarle a reconocerse así mismo para abrir la posibilidad de reconocer al otro y de esta forma aprender e interactuar juntos, construyendo procesos que les sean significativos y que contribuyan a sus aprendizajes; para ello, las experiencias son la principal fuente. Ahora bien, frente a las experiencias desde la filosofía como arte de vivir, Hansen (2013) expresa que "una de las tareas de la educación es ser conscientes de esa condición existencial y trabajar para hacer que los contactos de uno con el mundo sean tan fructíferos como las circunstancias permitan" (p.69).

Además, a partir de dichas experiencias los actores de la educación deben hallar un sentido de estabilidad, concebido como "una disposición o una sensibilidad que nos empuja a pensar en el cambio y en las fuerzas que lo desencadenan, en vez de, sencillamente, amilanarnos o retirarnos cuando nos enfrentamos a él" (Hansel, 2013, p.68). Dicha estabilidad permite a los seres ser puertas abiertas, cruzadas por caminos 
llenos de costumbres, de lo reiterativo, lo predeterminado y lo estable para llegar a otros lados donde se originen nuevos aprendizajes, los cuales tienen la posibilidad de ser o no adquiridos y aprendidos, dependiendo del interés, las ideas enraizadas desde la cuna $\mathrm{y}$ especialmente de las experiencias vividas.

Al pensar en otras escuelas, es preciso ser consiente que no siempre estas obtienen los resultados esperados, ya sea porque se desconoce su proceso en general, no se tienen los recursos suficientes para la implementación o simplemente se llega a un aula de la cual no se tiene conocimiento, como le sucede a los maestros especialmente rurales con Escuela Nueva que desconocen mucho del modelo $\mathrm{o}$ modelos existentes y no tienen las herramientas para abordar estas escuelas, como dice Beltrán y Cuellar (2014), "aquí se pone de presente un modo para juzgar al maestro como un experimentador desprovisto de las herramientas necesarias para aplicar coherentemente las modernas teorías pedagógicas" (p. 170).

En fin, repensar la educación traspasa el simple hecho de adoptar un modelo y generalizarlo, sin hacerle seguimiento o evaluar su impacto. Hacer conjeturas sobre la educación implica reconocer los aciertos de las otras escuelas y experimentar alrededor de modelos cercanos a nuestros contextos o de similares condiciones, para enriquecer esta experiencia, mientras se abre la posibilidad de explorar esas otras escuelas que aún no se reconocen, y en este ejercicio importa el hecho de admitir la existencia de un pensamiento complejo, mostrado por Morin (2011) como un pensamiento capaz de no quedarse encerrado en lo local y lo particular sino de concebir los conjuntos sería apto para favorecer el sentido de la responsabilidad y de la ciudadanía.

Profesor desde una visión cosmopolita

Para Hansel (2013), el

cosmopolitismo describe un espíritu de apertura a aprender de los contactos de la vida, especialmente de aquellos que son nuevos y por esa razón, pueden parecer extraños, inestables, inquietantes o quizás, incluso atemorizantes. Por tal razón, pensar en un profesor cosmopolita, es pensar una persona en constante reflexión, que recapacita en sí mismo y desde sí, en los demás, para replantearse valores y bases morales que transmitirá a sus estudiantes a partir de sus acciones. Así pues, los profesores son los sujetos que primero deben contemplar con otros ojos la educación para transformarla, haciéndola parte de su vida y logrando con esta, satisfacer necesidades propias que promuevan espacios ricos en aprendizajes significativos, recreen sus experiencias e inviten a los demás a dar ese pequeño paso de cambio; ya que,

Son los docentes quienes pueden dar, en primer lugar, un impulso a los procesos de transformación, a la investigación, a la innovación, a los cambios políticos, asumiendo el papel de "intelectuales transformadores" $y$ "actores ético- sociales" empeñados en conseguir un modelo de ciudadanía responsable 
ante la humanidad y el planeta. (Sánchez y García. 2017, p. 5)

De esta manera se va dando forma a una educación cosmopolita, en donde es conveniente que los maestros en formación o futuros profesionales en el campo educativo, sean conscientes de su labor como agentes de emancipación, que piensen cómo en su realidad, lograr enfrentar retos a partir del uso de nuevas herramientas, procesos y elementos que tengan a su disposición; puesto que para Hansel (2013), "las acciones de cambio inevitable, el cultivo de respuestas sobre el mismo y la posición crítica frene a los puntos de vista y conducta humana, son condiciones que subyacen en el quehacer de los profesores en cualquier escenario" (p.66).

Desde otra mirada, un proceso cosmopolita transformador, según Boni (2011), "nace del encuentro con lo culturalmente diferente, a partir del entendimiento y la práctica de una transculturalidad real que asume que no existen jerarquías entre las culturas" (p. 80). Por lo tanto, los docentes desde su formación están llamados a propiciar espacios de socialización de experiencias, mantener contacto con otros docentes y permitirse conocer el trabajo del otro como medio para reconocer, reconstruir o mejorar sus prácticas en beneficio de sus estudiantes, mostrándoles la importancia de las interacciones, los valores y el intercambio de conocimiento, como forma de reconocer transformaciones $\mathrm{y}$ apretura frente a lo que ha sido influenciado por la crítica.

En esta medida, la labor docente puede ser pensada no solo en función de enseñar conocimiento aislados, sino en servicio a los sujetos y todo cuanto les rodea, para hacer que el ser humano conciba su identidad, cultive la solidaridad y la fraternidad como parte de su humanidad, consiga desafiar incertidumbres y sea capaz de proponer soluciones a situaciones de su vida cotidiana, con el propósito de unir lo científico con lo humano y lo tecnológico con el desarrollo natural (Hessel y Morin, 2012). De esta manera, el docente auspicia una verdadera reforma de pensamiento que le acerca a la complejidad, donde se vislumbra la transdisciplinariedad como una solución para apropiar dicho pensamiento complejo, en función de propuestas innovadoras que apunten no solo al reconocimiento de saberes científicos sino de cosmovisiones y saberes tradicionales que se integren a proceso de investigación para pensar otras formas de educar.

\section{En busca de sociedades más justas: proyecto pedagógico de educación popular}

...el aprendizaje no tiene que ver con la memorización del conocimiento muerto y de las habilidades asociadas con el aprendizaje para un examen, sino con el compromiso con una lucha más amplia por los derechos individuales y la justicia social. (Giroux, 2018, p. 164)

\section{El porqué de la educación popular}

La problemática histórica del pueblo latinoamericano condicionada por los fenómenos de colonización, dominación, exclusión, marginación, opresión, desigualdad, injusticia e instrumentalización, producto de la 
imposición del pensamiento utilitario euro centrista y de la pragmática dominadora norteamericana, conduce a diseñar, construir e implementar una acción educativa con identidad auténtica, local, autónoma, liberadora y fundada en el respeto por las diferencias, en y desde el Sur y desde abajo, conocida como "Educación Popular", así lo afirma Mejía (2014) al señalar que,

La educación popular se sigue constituyendo como una apuesta por transformar la sociedad desde la educación, y en la urgencia de sus desarrollos $\mathrm{y}$ en la particularidad de los contextos y ámbitos brotarán otras tensiones que cada uno de los lectores de este texto debe complementar para mostrar la vitalidad de este pensamiento (p. 28).

Así mismo, se destaca la Educación Popular como una propuesta política-pedagógica, con un acumulado propio y un desarrollo desde distintos enfoques (histórico, epistemológico, sociológico, antropológico, conceptual y metodológico), cuya finalidad es la reconstrucción de un proyecto educativo y pedagógico para todos, orientado al establecimiento de una sociedad más justa, digna, humana y equitativa en un mundo globalizado y dominado por el capitalismo. Con relación a lo anterior, Muñoz (2013) califica la educación popular como "un proceso social mediante el cual los sectores populares llegan a convertirse en el sujeto histórico - mesiánico, gestor y protagonista de un proyecto liberador que encarne sus propios intereses de clase" (p. 158), del cual se espera se susciten espacios de reflexión y crítica ante los fenómenos que afectan al individuo y a los grupos, para pensarse en uno solo y hacer posible surgir nuevos enfoques, teorías y metodologías que permitan desvirtuar acciones de sometimiento individual o social.

Desde otra mirada, De la Calle (2011) indica que "la educación popular ha sido más explícita en el reclamo y la denuncia, en la intencionalidad y la conciencia de la deliberación de la acción educativa transformadora" (p.141); por ello la Educación Popular, vinculada con nuevas propuestas político-pedagógicas pensadas desde el Sur o desde Latinoamérica (Educación Liberadora, Pedagogía del Oprimido, Educación Emancipadora, Pedagogías crítico-sociales,

Pedagogías Comunitarias, etc.), tiene su punto de partida en la lectura crítica y propositiva de nuestra realidad, reconociendo la práctica educativa como agente para la transformación y superación de las condiciones que impiden que los pueblos del Sur tengan su autonomía, muestren su rostro oculto y levanten su voz silenciada, piensen por sí mismos $y$ decidan soberanamente $\sin$ las imposiciones de otras ideologías.

Esta pertinente acción educativa cuenta con un acumulado propio (hecho movimiento y propuesta político-pedagógica) y con alternativas educativas, políticas, culturales, sociales y económicas que reflejan los intereses y anhelos de los grupos populares del Sur como espacio geográfico y cognitivo. Dicho acumulado responde, 
entre otras, a las preguntas de la educación en dónde, para qué, para quién y desde dónde; además, dispone de nuevos escenarios, posee identidad $\mathrm{y}$ contenido que van surgiendo en el contexto, guiando a los sujetos a pensar no solo como individuos, sino en colectivo, a construir su identidad desde la toma de conciencia del ser de grupo, a tener sentido de pertenencia, compartir aprendizajes sociales, entre otras acciones en torno a la identidad. En este sentido, Lorenzo (2008) expresa que "el proceso complejo y dinámico de formación identitaria y cultural depende de la posibilidad de ese sujeto de pertenecer y de ser, en la medida en que se relaciona con los otros creando nuevos sentidos de pertenencia y de referencia" (p. 35). En esta medida, la creación de identidad favorece los procesos populares, donde se reconoce el ser como parte de un todo, promueve beneficios comunes $\mathrm{y}$ da paso a construcciones colectivas que propenden justicia, equidad y emancipación.

Otro de los ámbitos que circunda la Educación Popular, es la preocupación por la naturaleza y la importancia de hallar un equilibrio entre la relación humano-naturaleza, a partir de lo que Mejía (2014) denomina eco-política, "como un asunto de los centrales y un componente básico de cualquier transformación" (p. 12); esta eco-política, invita a pensar en los usos y connotaciones que se le están dando a la naturaleza y las diferentes formas de vida que la habitan, preocupándose por mantenerla viva y rescatarla de los ataques que sufre bajo los rostros del desarrollo, la tecnología y la sustentabilidad, los cuales deben ser un solo engranaje que se piense en torno al bienestar de todos y no de unos pocos que lideran procesos globalizantes y capitalistas.

Así pues, la Educación Popular promueve la recuperación de cosmovisiones que protegen el medio ambiente y busca fortalecerlas en modelos sociales como el Buen Vivir, que ha ido incorporando construcciones en función de la igualdad, la solidaridad y la protección de la naturaleza y todo lo que en ella se forma; buscando así, una interacción dinámica y sinérgica con el entorno natural, en perfecta armonía con la madre tierra y su cuidado.

También, cabe resaltar que otro objetivo de la Educación Popular es refundar una democracia genuina desde la identidad de una sociedad que siente, piensa y sistematiza su realidad desde el Sur y desde abajo, lugar donde es, está y existe en el mundo. Una educación así concebida desde el Sur y desde abajo, será el instrumento liberador para el surgimiento de una subjetividad rebelde que, con un nuevo dispositivo metodológico, conceptual y epistemológico, logre romper las cadenas que nos esclavizan, mediante la acción transformadora y emancipadora que coloca a nuestro alcance esa propuesta político-pedagógica.

\section{Saberes que se extienden sin frontera}

La Educación Popular, como acción educativa y pedagógica comprometidaconelserlatinoamericano y situada en un contexto diverso y pluridimensional del Sur, también se enriquece con elementos políticos, sociales, culturales, conceptuales, 
metodológicos y económicos propios de nuestros pueblos ancestrales, con sus cosmogonías y cosmovisiones que sirven de fundamento para el diseño, la construcción e implementación de una propuesta político-pedagógica liberadora desde el Sur y desde abajo; pero que no solo se quede en el Sur, sino que se extienda, se confronte con otras propuestas y se nutra de ellas, a partir de diálogos de concertación entre saberes y conocimientos, en procura de invalidar pensamientos unilaterales de una sociedad basada en meras dicotomías que suscitan la segregación generada entre las potencias dominantes y los pueblos dominados.

En este sentido, esta propuesta multidimensional se vislumbra como una acción pedagógica de negociación cultural, en cuyos ámbitos de individuación, socialización, vinculación a lo público, a movimientos y formas organizadas, construcción de procesos de gobernabilidad y masividad, se hace específico el proceso de diálogo de saberes (intraculturalidad), confrontación de saberes (interculturalidad) y negociación cultural (transculturalidad) (Mejía, 2014, p. 11). Dicho diálogo y confrontación, se establece desde lo que Mejía (2014) denomina el principio de complementariedad, donde la interculturalidad y la transculturalidad posibilitan pensamientos universales alrededor de la concertación de acciones críticas que logren emancipar la sociedad de ideas que la reprime.

De la misma manera, la propuesta de Educación Popular permite cerrar brechas entre las fronteras del Sur y el Norte, a partir de una relación de identidades que distingue necesidades y oportunidades en común y se enriquecen a través de un aprendizaje dialógico que modifica realidades sociales sesgadas y divididas por ideologías o cosmovisiones distantes; de manera que estas últimas, pueden dialogar para que confluyan en concepciones comunes alrededor del conocimiento, la cultural, las estructuras sociales y la educación, dando paso a otros mundos posibles de todos y para todos. (Mejía, 2014)

A lo anterior, Mejía (2014) añade que el diálogo de saberes y conocimientos abren caminos al pensamiento hegemónico en lo cultural y pedagógico, lo cual rompe con los sistemas cerrados. En esta perspectiva, la complementariedad otorga múltiples posibilidades de debate y concertación, donde la educación popular provee de técnicas, métodos y estrategias a la sociedad para modelarlos en espacios dinámicos, cambiantes y fluctuantes, invitando a los sujetos a ser actores de intercambio de saberes generados a partir de estas prácticas para que a la vez se motiven a comprobar, ajustar o modificar las mismas a partir de procesos creativos; ya que el llamado de la Educación es a "imaginar, inventar e instaurar cambios en las técnicas de enseñanza usadas y la forma como se conoce y experimenta la realidad, con una actitud de afecto y reconocimiento recíproco" (Berbesi, 2014, p. 262), en donde ese reconocimiento recíproco se abre desde el Sur hacia el Norte y viceversa.

En suma, se puede afirmar que el intercambio de saberes y conocimientos entre sur y norte a partir del análisis de las cosmogonías y las ciencias 
modernas como complemento uno de la otra para generar nuevas teorías en beneficios globales y no solo locales, permite construir un saber de frontera, en donde también habrá que pensar las formas institucionales que pueden cobijar no solo ese intercambio, sino también las formas bajo las cuales va a ser posible lograr esa producción de saber cómo lo expone Mejía (2014).

Los educadores populares como agentes de cambio

Los educadores populares están llamados a efectuar un despliegue de pilares, enfoques, métodos, conocimientos y estrategias que emergen desde la Educación Popular, partiendo del lugar donde se desarrollan los sujetos; porque allí, pueden favorecer los aprendizajes significativos y la construcción de identidad, bajo la lógica de un espacio que reconocen como suyo. En este sentido, Lorenzo (2008) manifiesta que,

Cuanto más articulada esté la práctica pedagógica con la experiencia y la vivencia de los ámbitos en los que los sujetos se forman -es decir, familia, barrio, comunidad, instituciones, espacios y grupos humanos formales e informales, etc.-, mayores posibilidades encontrará el reconocimiento de la identidad popular y cultural de cada individuo, grupo social o comunidad (p. 39).

En este ámbito, "el contexto es un elemento clave para ser aprovechado por el educador popular en busca de la emancipación humana y la descolonización del ser" (Muñoz,
2013, p.161), dando al educando la posibilidad de reconocer lo que sabe y ejecutar procesos de metacognición a partir de ejercicios de diálogo de saberes y conocimientos que se promuevan en el aula o el lugar donde se aprende. En esta línea, ser educador popular implica pensar alrededor de cambios que generen actos equitativos, favorezcan los grupos excluidos, dominados y segregados y favorezca en los estudiantes el reconocimiento de su identidad a nivel individual o grupal; además de "pensar esas otras formas de la democracia y la ciudadanía para hacerla más horizontal, y que son visibilizados por nuestros grupos populares, más allá de quienes han terminado rescatando y trasladando a nuestros contextos la democracia radical" (Mejía, 2014, p. 16).

Para De la Calle (2011), "los educadores populares pretenden la formación del sujeto humano en términos de un aprendizaje social potencializador de la constitución ciudadana, en busca de armonizar los comportamientos democráticos con las perspectivas de los derechos singulares" (p. 140); dicho de otra forma, los sujetos formados desde la Educación Popular tienen la capacidad de tomar conciencia alrededor del bien común, no solo local, sino global, mediado por la participación, el respeto por el otro y sus ideas y especialmente de utilizar lo aprendido para favorecer e impulsar el desarrollo de su comunidad. Para lograr lo anterior, Berbesi (2014) expresa que:

El proceso educativo deberá ser coherente, que los capacite para vivir en dignidad y ejerza sus 
derechos de ciudadanía, en respuesta a las necesidades humanas, capaces de construir la historia en colectivo, orientadas a leer la realidad, formando seres solidarios y darles las herramientas para que subsane problemas y tomen decisiones (p. 265)

Otro aspecto relevante del educador popular, según Mejía (2014), es que genera procesos de producción de conocimientos, saberes y de vida con sentido para la emancipación humana y social, donde, a partir de la sistematización de aquellos saberes hasta el momento sometidos y poco reconocidos como las cosmogonías y los no científicos, se validan por medio de investigaciones, originando otras teorías o métodos que llevan a aplicar y visibilizar estos saberes en diferentes campos de acción humana individual y social. Para apoyar dicha sistematización, la Educación Popular "se ve jalada a generar procesos pedagógicos específicos para los que se generan en el ciber-espacio, en las redes, en las wikis, y que transforman el espectro y las formas de lo masivo en este siglo XXI" (Mejía, 2014, p. 22). Así, la educación popular toma aspectos de la tecnología que le sirven para promover y hacer más accesibles sus prácticas, $\mathrm{y}$ a la vez analiza la tecnología desde una mirada crítica, para que su uso sea en favor de la humanidad y no en contra.

De esta forma se resalta que la formación de autonomía es un eje de la Educación Popular, en tanto educador como educando reafirman su identidad, el liderazgo y la toma de decisiones; dicha autonomía también comprende dejar que los miembros de la comunidad educativa logren aprender a aprender, constituyéndose como sujetos críticos, libres y conscientes de la construcción de un proyecto de vida en los que la introspección y extrospección, les permite la reflexión sobre su identidad. (Berbesí, 2014).

\section{La complejidad y sus elementos para la educación}

Tenemos que confeccionar o construir entre todos nuevos trajes, nuevas formas de vestir la educación para que responda, realmente, a las necesidades de una sociedad compleja

(Pozo, 2020, p. 18)

\section{Educación y complejidad}

La educación ha sido vista desde tiempos remotos como la base para el cambio, el desarrollo y un escenario de interacciones, donde las ideas confluyen en pro de la sociedad. A pesar de ello, ha sido afectada por las diferentes epistemologías y paradigmas crecientes de las distintas épocas. Por ejemplo, con la epistemología positivista emergió el concepto de sujeto y objetividad, en los cuales la acción de conocer, que es dinámica, de intercambios y multidimensional, se encerró en el objeto del conocimiento, dando paso a un sujeto que razona desligado de sus sentimientos, emociones y sensaciones múltiples que produce el exterior para lograr la objetividad, concebida en una acción imparcial que revela solo la realidad sin estar permeada por la mirada individual.

Para Najmanovich (2008), "pensar un universo independiente del pensamiento que lo está pensando: 
he aquí la paradoja fundante de la epistemología"(p. 16); en otras palabras, el conocimiento no debe darse aislado entre sujeto y objeto; por ello, la educación como medio para explorarlo, ha de promover las interacciones y el establecimiento de relaciones no lineales entre el individuo y lo que se desea conocer, abriendo espacios para que en estos dos se vincule el contexto, las ideas externas y por supuesto, la mirada individual del sujeto frente a su objeto de conocimiento.

Con la idea anterior, se tiende a abandonar la estética del conocimiento llamada representacionalismo, caracterizada por Najmanovich (2008) como aquella que "solo admite mundos disjuntos, aislados, mutuamente excluyentes" (p. 16), es decir, la realidad es única al ojo humano, así el sujeto al no interactuar con el mundo externo, no lo logra ver; por tanto, solo se hace una representación de lo real, aislada de cualquier percepción sensorial que le puede llevar a reformular dicha realidad. En el campo educativo el representacionalismo se ha ido aislando para dar paso a nuevas formas de pensamiento, en las que el conocimiento no se origina de una sola forma según lo expresaba la ciencia clásica, por el contrario, este se enriquece con las múltiples visiones que le pueden brindar desde el exterior.

Así, la educación al abrir nuevas posibilidades de conocer y producir conocimiento, en la variedad de perspectivas que nos provee el contexto y en términos de multidimensionalidad, permite el ingreso al enfoque de la complejidad que, además de ser un componente de cambio que suscita la singularidad rechazando la aceptación de una visión única del mundo, "se acerca a la educación para abrir sus puertas a los aportes fundamentales de esta como lo son viabilizar otros interrogantes, gestar nuevas miradas sobre el mundo y sobre nosotros en él" (Najmanovich, 2008, p. 20). De esta manera, el acto de enseñar no queda resumido al ejercicio de transmitir y reproducir una misma acción frente al conocimiento; por el contrario, la enseñanza se dinamiza en tanto los actores (maestro-estudiante) comparten experiencias, aclaran inquietudes y construyen bajo la experiencia otras formas de pensar y conocer.

En esa línea de pensamiento lo proponen Arroyave y Barreto (2020),

La consideración del pensamiento complejo en un mundo que cambia de forma acelerada $y$ constante es una invitación a no dejarse llevar por el afán, contemplando a la educación como un asunto de producción en serie, $y$ abrir lugar a la reflexión y consciencia de los sujetos actores-autores educativos para llevar a cabo interacciones recíprocas modificantes con el otro, con el medio y hasta consigo mismo, si es que se quiere hacer un aporte significativo a la sociedad (p. 81)

Ahora bien, los enfoques de la complejidad conllevan aun pensamiento dinámico pensado bajo la configuración de las transformaciones en la realidad 
que afectan, generan caos y se abren para asimilar nuevos procesos, entrelazar teorías y se acerca a otros campos del conocimiento para alimentarlo y enriquecerlo. Dicho pensamiento dinámico debe ser llevado al proceso de enseñanza aprendizaje a partir de espacios de innovación que faciliten a los estudiantes ser protagonistas y constructores de su propio aprendizaje $\mathrm{y}$, en función de esta lógica, edifiquen un pensamiento dinámico que sea poiético, permitiéndoles producir, crear $\mathrm{y}$ transformar su entorno con nuevas vivencias que los acerquen a otros conocimientos. De ahí que surja la necesidad de replantear los currículos pensados para abordar los intereses de los estudiantes, los requerimientos del contexto y alejados de lo preestablecido como forma única de transmitir conocimiento.

En la misma línea, respecto de las relaciones como eje fundamental del pensamiento dinámico, cabe resaltar que estas dan origen a la dinámica vincular de la que emergen los sistemas complejos; por tanto, el sistema educativo se ve permeado de esta dinámica vincular, que es un componente que ofrece a los estudiantes herramientas para que logren entretejer conocimientos, adquirir habilidades que les faculta para sortear situaciones problemas, reconocer la realidad de su contexto en la diversidad, plantearse interrogantes, comprender la existencia del error y las múltiples visiones sobre la verdad. De esta manera, en palabras de De Jesús, et al., (2007):

Una educación desde la perspectiva compleja tiene que ser pertinente desde una visión planetaria, lo que se manifestaría en una propuesta para la comprensión y edificación del fenómeno educativo como algo más humano, multidimensional, integrador, intercultural, transdisciplinario, reconocedor del error, la incertidumbre y la diversidad $y$ de un conocimiento apto para el abordaje de problemas, para la formación de un ciudadano involucrado con las necesidades de su entorno desde un ejercicio de transformación permanente. (p. 3)

En coherencia con lo expresado por los autores, se vislumbran nuevos retos para la educación enmarcados en procesos de enseñanza aprendizaje, en los que se reconozcan saberes socialmente significativos que admitan no solo trasferir conocimientos, sino valores, costumbres y formas de actuar en concordancia con las necesidades de cada individuo y con las de los demás, manteniendo un equilibrio armónico entre seres humanos y naturaleza. Esto generará mayor conciencia planetaria e intercambio de ideas que modifiquen los esquemas comunes de desarrollo y busquen alternativas dentro de los sistemas complejos para hacer de la convivencia y el proceso de globalización dos ejercicios más humanos.

\subsection{Paradojas: un elemento para la educación}

Hacer uso de elementos 
complejos en la producción de conocimiento lleva a que el ser humano realice un ejercicio inagotable en el cual va comprobando, indagando, experimentado y advirtiendo sobre nuevos saberes, esto le hace a su vez responsable de transmitir dicho conocimiento como una bola de nieve que enriquece las maneras de conocer y a los saberes. En palabras de Najmanovich (2008), "la complejidad es una reconfiguración global de las formas de producir, validar y compartir el conocimiento" (p. 31); entonces los enfoques complejos usan diversos recursos para fortalecerse y entre ellos encontramos las paradojas.

Vega y Olmos (2011) indican que "un hecho se considera paradójico cuando resulta contrario a las expectativas razonables, rompiendo alguna regularidad real o presunta" (p. 443). Así pues, la paradoja se constituye en un elemento de la complejidad, en cuanto se sale de los conceptos lineales y permite hacer conjeturas sobre un concepto, teoría, ciencias u otro elemento que se aborde alrededor del conocimiento, como los expresa Najmanovich (2008) "cuando nos encontramos con una paradoja "chocamos" contra los límites de nuestro paisaje cognitivo, ya se trate de un paradigma, un modelo, una teoría, o una cosmovisión" (p. 19).

Se puede afirmar, que las paradojas son recursos que sirven en el proceso educativo, ya que constituyen respuestas e incertidumbres alrededor de lo que se enseña, cómo se enseña y por qué se enseña, permitiendo esto modular acciones que fortalezcan los procesos de enseñanza aprendizaje, de esquemas de pensamiento que llevan a los actores del proceso a cruzar límites, establecer semejanzas, salirse de la zona de confort y tocar fibras que se perciben más allá de la realidad que nos rodea. De esta manera se consideran las paradojas como "compuertas evolutivas que nos convidan a validar creencias, teorías y paradigmas, y para salir de ella construyendo paisajes cognitivos con variedad dimensional" (Najmanovich, 2008, p. 19).

Por otra parte, la educación se mueve en tendencias según teorías dadas a partir de procesos de investigación en contexto, sobre esta idea De Jesús et al., (2007) mencionan que "los Escépticos, precursores del Constructivismo sostenían que estamos atrapados en una paradoja porque queremos construir conocimiento verdadero y solo accedemos al conocimiento a partir de la experiencia y esta es cambiante, mutable" (p.4). Dicha paradoja es la de la educación, por esta razón, hay que evitar abordar los procesos de enseñanza en un contexto cambiante que le es distante para el estudiante; así, para que el contexto sea significativo, el estudiante tiene que experimentar en este, establecer vínculos de reconocimiento, exploración, indagación y percibir sus cambios para apropiar los nuevos saberes que le brinda a medida que pasa el tiempo y es permeado por distintos factores que le modifican.

Así mismo, al estar la educación en espacios dinámicos, cambiantes donde se generan nuevas visiones del mundo y de su contenido, Vega y Olmos (2011) reconocen que "las paradojas son un terreno fértil, donde 
se pone a prueba nuestra capacidad de buscar soluciones"(p. 444); por ello, se configuran en herramientas para fortalecer el razonamiento de los estudiantes, ofreciendo la posibilidad de cuestionarse por los elementos que circundan sus contextos, repensando sus formas de ser, actuar y convivir con los demás y de igual forma buscando múltiples soluciones a situaciones que convergen tras el análisis de cada elemento que se constituye a partir de una paradoja.

Desde otra mirada, la escuela puede llegar a convertirse en un espacio estático con ausencia de cambios, manteniendo una misma línea de acción que otorgue la razón a las políticas educativas preestablecidas y no contempla la diversidad para efectos de enseñar. Así pues, al pensar este hecho como paradoja del fin de la educación (como eje promotor de cambio), se puede usar esta como un medio para salir de dicho estado, ya que en referencia de Najmanovich (2008) "la paradoja nos provoca, y nos exige, pensar de otro modo para poder salir de lo que podría ser un círculo vicioso" (p. 19). En concordancia, dicha paradoja educativa puede conllevar a repensar cambios en la escuela convirtiéndola en un espacio de experimentación en la que se estimule la creatividad de los estudiantes, se piense en espacios que propendan por la cultura, el arte y el ambiente, se distingan como saberes indispensables para formación de ciudadanía.

De esta manera, se identifican las paradojas como un elemento para fortalecer la educación desde la visión de cambio, el fomento de la crítica en los docentes y estudiantes y una forma de robustecer la dialógica en las aulas, donde la circulación de saberes se fortalezca a partir de la interacción e intercambio de conocimientos, para dar fin a paradojas existentes con acciones novedosas y a su vez originando otras que permitan estar dentro de un círculo de constante cambio y reflexión frente a las acciones que se están dando en la escuela.

\section{Importancia de las redes en educación}

Hoy día, las relaciones mueven las dinámicas del mundo y en tendencia está la organización de grupos sociales, estudiantiles, laborales y de diferentes de personas con intereses comunes. A estas asociaciones se les conoce como redes, este concepto es abordado por Najmanovich (2007), quien asegura que "pensar en red implica ante todo la posibilidad de tener en cuenta el alto grado de interconexión de los fenómenos y establecer itinerarios de conocimiento tomando en cuenta las diversas formas de experiencia humana y sus múltiples articulaciones" (p. 73). Con esto, se establece una nueva línea de interacción entre las personas, otras formas de aprender, expresarse y adaptarse a nuevas posibilidades que se crean a partir del compartir de experiencias, de nuevos saberes y de la construcción colectiva de conclusiones o constructos que conllevan a un beneficio común.

Por lo anterior, la educación viene avanzando también en la creación de redes que permiten ampliar el campo de acción de los estudiantes alrededor de los conocimientos y aprendizajes deseados por ellos, posibilitando nuevas 
formas de aprender, generar círculos de saberes y, especialmente, fortalecer un saber en torno al gusto o habilidad de quien desea dominarlo y enriquecerlo con el aporte de los demás. Además, las redes configuradas y organizadas por gusto, necesidad, curiosidad o interés propio, permite a sus integrantes abrirse a nuevas posibilidades, expresar sus ideas sin temores a señalamientos o prejuicios, lo que confiere a la red una característica de libertad que permite avanzar a espacios poco explorados y hacerlos propios para reconstruirlos a partir del interconocimiento.

Najmanovich (2007) comenta que "las redes son de naturaleza heterárquica y adhocrática, puesto que toda configuración es un resultado adhoc de los encuentros"(p. 77); por ello, las redes no limitan, no se constituyen en un contrato para aquellos que forman parte de estas, lo que las hace libres, únicas y especialmente posibles de acceso a quienes tengan interés en ellas. En concordancia con esto, Salcedo (2014) enuncia:

Jóvenes interconectados, sin fórmulas, sin jerarquías, desde relaciones horizontales, sin el tiempo como camisa de fuerza, sin la necesidad de los encuentros para el debate y la toma de decisiones, trabajan colectivamente sin desconocer lo individual. Se anexan y se retiran del proyecto cuando desean, pues su motivación no viene del líder, sino de sí mismo y de su fe en lo que está haciendo. El sujeto está desarrollando plenamente sus habilidades, aporta lo que sabe, no lo que le pide la institución y el equipo. Y algo que valoramos mucho los humanos, en este trabajo en red se nota en el acto tu aporte. (p. 7)

Así mismo, las redes en el campo educativo se convierten en un espacio único, que oferta conocimiento y lo dinamiza a través de las emociones e intercambios de sus miembros, abriendo la posibilidad de que estos amplíen su visión del mundo en retrospectiva, verificando aportes y proyectando nuevos, en miradas amplificadas que enfoquen no solo la realidad cercana, sino de paso a hacer una inspección más profunda como la que propone Najmanovich (2008) con el "configuraZoom" donde especifica que este "admite el ajuste de varios parámetros simultáneamente posibilitando un sistema de enfoque multidimensional" (p. 32). Dicho de otra manera, él configura - Zoom, admite ampliar el conocimiento tras la experimentación, la multidimensionalidad, el cambio de visión singular para transformarla en plural, ante la concepción de conjunto como elemento para la construcción de nuevas ideas, teorías, proyectos $\mathrm{y}$ transformación que se forjan en red.

De esta forma se logra entender la idea de red como un todo que cualifica, dota de importancia y reconoce la labor de sus partes, lo que motiva a sus miembros a estar en ella y reconoce, en palabras Vilar (2008), que "la red es en sí misma un objeto de reflexión, investigación y estudio, al margen de las problemáticas que quiera abordar, por lo que requiere un importante esfuerzo de metacognición" 
(p. 271). Como se aprecia las relaciones generadas en un colectivo ya sea educativo, social, político se solidifican en la red dinámica para construcción de conocimiento y servicio mutuo en pro del sujeto y objeto de conocimiento.

\section{Conclusiones}

La educación es un proceso en el que influyen diversos factores, entre ellos el currículo, elemento esencial para organizar los procesos de enseñanza aprendizajes, pensados desde las necesidades del contexto y que hace partícipes a todos los miembros de la comunidad educativa en su continua construcción y reconstrucción. Dicho currículo debe ser leído y apropiado por docentes cosmopolitas, en quienes predomina la reflexión continua sobre la realidad educativa y su práctica, con el fin de favorecer los intereses de aprendizaje de los estudiantes para lograr transformar sus realidades por diversas que sean. Así mismo, el currículo como facilitador y mediador de procesos, logra su autonomía tras la implementación y evaluación de modelos pedagógicos que afecten los contextos, se permitan pensar en lo que requieren que se les enseñe a los estudiantes y cómo hacerlo, de manera que no se limite a propuestas únicas o estandarizadas, lo cual le permite estar dispuesto al cambio a mediada que la sociedad y el contexto también cambian.

Como acción educativa
auténtica, que busca responder
a problemáticas del pueblo latinoamericano, condicionadas por fenómenos como la colonización, la migración, las injusticias sociales, el capitalismo acaparador y la opresión entre otras, la Educación Popular busca transformar la sociedad desde la educación. Para ello se concibe como una propuesta político-pedagógica, que suscitan espacios de reflexión y crítica sobre la sociedad, de los cuales se generan nuevos enfoques, teorías o métodos, en pro de fundar una democracia genuina y un equilibrio entre hombre y naturaleza pensado desde el buen vivir. De otra parte, la educación popular demanda de docentes que articulen sus prácticas al contexto donde se desarrollan los educandos, pensando en cambios que generen equidad y promuevan la formación autónoma, no solo de estudiantes sino de los mismos docentes. De esta manera, la Educación Popular rompe con sistemas cerrados, a partir del diálogo entre saberes y conocimientos que se confrontan desde diferentes posturas y contextos locales y globales.

La complejidad y sus elementos han influenciado al proceso educativo, viabilizando la construcción de conocimiento desde las interacciones y relaciones no lineales entre el individuo y su objeto cognoscente. De esta manera, la educación se dinamiza a partir del diálogo activo de saberes entre sus actores, donde se destaca al estudiante como protagonista en el proceso de aprendizaje brindado en aulas que promueven la innovación y la formación no solo desde el conocimiento, sino en el ser ético, social, político y humano. Otro escenario posible para la educación como sistema complejo, es el uso de las paradojas, elemento que le permite repensarse en torno a respuestas e incertidumbre sobre las acciones generadas alrededor de lo que 
se enseña y como se enseña. También, en un sistema educativo circundado por la complejidad, se hace importante abrirse a las múltiples posibilidades que le otorga el contexto y para ello una forma dinámica de hacerlo es el trabajo en red, donde se construyen, fortalecen y generan diferentes acciones propuestas en grupos con propósitos semejantes.

En suma, la educación está pensada como un espacio donde priman las interacciones entre los actores del proceso con el contexto para generar aprendizajes más significativos; así mismo, existen diferentes epistemologías, teorías, paradigmas $\mathrm{y}$ propuestas que han cerrado o diversificado las acciones implementadas por las instituciones educativas; por tanto es deber de estas y su comunidad en general verificar la pertinencia de dichas propuestas en favor de los contextos y su desarrollo desde una lectura crítica y reflexiva permanente, es pues un transitar hacia "la oportunidad y la responsabilidad de trabajar por una educación mejorada y, a la vez, transformadora. Una educación en la que no solo se aprenda bien, sino que también sea útil para vivir mejor" (Musons, 2021, p. 15).

\section{Referencia bibliográfica}

Barreto Cruz, M. A., \& Arroyave Giraldo, D. I. (2020). Educando desde la paradoja: un desafío frente a un contexto donde lo más estable es el cambio”. En D. I. Arroyave Giraldo (Ed.), Estudios multirreferenciales sobre educación y currículo, lecturas desde la complejidad. (pp. 61-82). Editorial Bonaventuriana.
Beltrán, R. R., \& Cuellar, M. Y. C. (2014). La modernización de los contenidos y métodos de enseñanza: reflexiones sobre la Escuela Nueva en Colombia. Revista historia de la educación latinoamericana, 16(22), 157-172.

Berbesí, N. C. G. (2014). La educación popular y las acciones pedagógicas vinculantes. Educere, 18(60), 257267.

Boni Aristizábal, A. (2011). Educación para la ciudadanía global. Significados y espacios para un cosmopolitismo transformador. Revista Española de Educación Comparada. (17):65-85. http://hdl. handle.net/10251/45824

Contreras D. J. (2010). Otras escuelas, otra educación, otra forma de pensar en el currículum. En: Saberes e incertidumbre sobre el currículum. Editorial Morata, Pág. 548 - 566

De Jesús, M. I., Andrade, R., Martínez, D. R., \& Méndez, R. (2007). Repensando la educación desde la complejidad. Polis. Revista Latinoamericana, (16). URL: http://journals.openedition.org/ polis/4581

De la Calle, C. V. (2011). La educación social y popular en Colombia. Relaciones y búsquedas: treinta años de legitimidad. Revista Cientifica Guillermo de Ockham, 9(1), 133-146.

Giraldo, E., Cadavid, A. M. y Flórez, S. (2019). Posibilidad de acuerdos sobre las concepciones de currículo para la formación de maestros. Educación y Educadores 22(1), 9-22. DOI: 10.5294/ edu.2019.22.1.1

Giroux Henry, (2018). Manifiesto por una pedagogía crítica. En Aparici et al., LA OTRA EDUCACION, pedagogías críticas para el siglo 
XXI. (pp. 155-169). Universidad Nacional de Educación a Distancia -UNED-.

Hansen, David T. (2013). El profesor cosmopolita en un mundo global: buscando el equilibrio entre la apertura a lo nuevo y la lealtad a lo conocido. Narcea.

Hessel, S. y Morin, E. (2012). El camino de la esperanza. Una llamada a la movilización cívica. Epub. P. 2731

Lorenzo, Z. B. (2008). Educación popular, cultura e identidad desde la perspectiva de Paulo Freire. Obtenido de CLACSO: http:// biblioteca. clacso. edu. ar/ar/libros/ campus/freire/06Brito. pdf.

Mejía, M.R. J. (2014). La Educación Popular: Una construcción colectiva desde el Sur y desde abajo. Arquivos Analíticos de Políticas Educativas, 22 (62).

Morín E. (2020). Los desafíos del poscoronavirus. En Cambiemos de vía, lecciones de la pandemia. (p. 38-48), Planeta.

Morin, E. (2002). La cabeza bien puesta. Repensar la reforma. Reformar el pensamiento. Nueva visión.

Morin, Edgar. (2011) La vía para el futuro de la humanidad. Paidós.

Muñoz Gaviria, D. (2013). Lectura de contexto: La educación popular como práctica libertaria. El Ágora USB, 13(1), 155-162

Musons Jordi, (2021). Reinventar la escuela: Una brújula para familias y educadores para comprender la educación del siglo XXI. Arpa.

Najmanovich, D. (2007) El desafío de la Complejidad: Redes, cartografías dinámicas y mundos implicados. Utopía y Praxis Latinoamericana, vol. 12 , núm. 38 , pp. $71-82$

Najmanovich, D. (2008). Mirar con nuevos ojos nuevos paradigmas en la ciencia y pensamiento complejo. Editorial Biblos. Pág. 15-33

Porlán Rafael, (2020). El cambio de la enseñanza y el aprendizaje en tiempos de pandemia. Revista de Educación Ambiental y Sostenibilidad2(1), 1502. doi:10.25267/Rev educ ambient sostenibilidad.2020.v2.i 1.1502

Pozo Juan Ignacio, (2020). La educación esta desnuda: Lo que deberíamos aprender de la escuela confinada. SM.

Salcedo, H. (2014). Paradojas de la Educación actual. Congreso Iberoamericano de Ciencia, Tecnología, Innovación y Educación, universidad Autónoma Latinoamericana.

Sánchez, M. C. H., y García, B. G. (2017). Currículum y práctica docente: hacia una educación transformadora. Congreso Nacional de Investigación educativa- COMIE.

Vega, L., \& Olmos, P. (2011). Compendio de lógica, argumentación y retórica. Editorial Trotta. Pag 443444

Vilar, J. (2008). Implicaciones éticas del trabajo en red y la acción comunitaria. Cultura $y$ Educación, 20(3), 267-277. 